\title{
Foco de Doença de Chagas em Arcádia, Estado do Rio de Janeiro, Brasil
}

\author{
Elias Seixas Lorosa ${ }^{+}$, Márcio Valério Monteiro Pinto Valente, Vanda Cunha, \\ Herman Lent, José Jurberg
}

\begin{abstract}
Laboratório Nacional e Internacional de Referência em Taxonomia de Triatomíneos, Departamento de Entomologia, Instituto Oswaldo Cruz-Fiocruz, Av. Brasil 4365, 21045-900 Rio de Janeiro, RJ, Brasil
\end{abstract}

Source of Chagas Disease in Arcádia, State of Rio de Janeiro, Brazil - Following the report of triatomine nymphs in a house in Arcadia, Miguel Pereira, state of Rio de Janeiro, Brazil, the infested dwelling was checked. Several eggs and 46 specimens of Triatoma vitticeps (Stal, 1859) were collected. Among them, adults and nymphal instars accounted for $43.5 \%$ and $56.5 \%$, respectively. Analysis of blood meals showed the ecletism of this species; 24 (52.2\%) were single feeds, 18 insects (39.1\%) fed on two hosts and 4 (8.7\%) on three hosts. Trypanosoma cruzi infection rate of examined specimens was $13 \%$. Finally one of the residents of the house was positive for anti-T. cruzi antibodies using indirect immunofluorescence.

Key words: Triatoma vitticeps - Hemiptera - Reduviidae - precipitin test - Chagas disease - Rio de Janeiro - Brazil

Seguindo relatório de encontro de ninfas de triatomíneos em uma casa em Arcádia, Miguel Pereira, estado do Rio de Janeiro, Brasil, resolvemos investigar o domicílio infestado. Neste local coletamos vários ovos e 46 espécimes de Triatoma vitticeps (Stal, 1859). Dentre eles, adultos e estádios ninfais apresentaram, respectivamente, $43,5 \%$ e $56,5 \%$ de representatividade. Através da análise do conteúdo estomacal pela técnica de precipitina constatamos o ecletismo alimentar desta espécie 24 $(52,2 \%)$ apresentaram repastos sangüíneos provenientes de uma só fonte, $18(39,1 \%)$ de duas fontes e quatro $(8,75 \%)$ de três fontes. A infecção natural por Trypanosoma cruzi foi detectada em seis espécimes representando 13\% da amostra total. Foi constatado, através do teste de imunoflorescência indireta para anticorpos anti-T. cruzi, que um dos moradores da casa apresentou reação positiva.

Em junho de 2002, o Laboratório Nacional e Internacional de Referência em Taxonomia de Triatomíneos recebeu para identificação alguns exemplares de triatomíneos coletados em um domicílio localizado em Arcádia, fazenda Terra Fria, distante $30 \mathrm{~km}$ da cidade de Miguel Pereira, a $900 \mathrm{~m}$ de altitude sobre o nível do mar.

Os insetos foram identificados como T. vitticeps, uma espécie de hábitos silvestres, esporadicamente encontrada dentro do domicílio; quando adultos são atraídos pela luz em casas próximas às matas (Lent 1942, Gonçalves et al. 1998).

No estado do Espírito Santo, esta espécie foi encontrada domiciliada (Santos 1969) com a suspeita de que

Com auxílio do CNPq, Faperj e Convênio 08/2003 Fiocruz/ Funasa

+Autor de contato. Fax: +55-21-2573.4468. E-mail lorosa@ioc.fiocruz.br

Recebido em 16 de abril de 2003

Aceito em 10 de setembro de 2003 possa transmitir a doença de Chagas, em particular no município de Alfredo Chaves (Lent 1942, Pinto 1969). Dois trabalhos assinalaram a alta dispersão do T. vitticeps no estado do Espírito Santo e chamaram atenção para a alta taxa de infecção natural por T. cruzi em todo o estado, oscilando entre $25 \%$ a $64 \%$ de positividade (Silveira et al. 1983, Sessa \& Carias 1986). Por outro lado, a prevalência da infecção humana por T. cruzi tem se apresentado sempre baixa em inquéritos realizados nessa região, seja na população geral, entre escolares ou em bancos de sangue (Barros et al. 1975, 1980). Esporadicamente, relatam-se casos agudos no estado do Espírito Santo com provável contaminação através de vetor ou por transmissão congênita (Pinto et al. 1986). No inquérito sorológico levado a cabo entre 1978 e 1980 encontrou-se uma prevalência de $0,32 \%$ entre a população geral de áreas rurais deste estado (Camargo et al. 1984).

T. vitticeps é bem conhecida através de vários pontos de vista, tanto morfológicos como biológicos. As ninfas, os ovos e os folículos testiculares das ninfas macho de $5^{\circ}$ estádio foram estudados por Jurberg e Campos (1995). Os adultos foram descritos por Lent e Wygodzinsky (1979) e a genitália do macho está representada em suas estruturas através de um estudo comparativo entre seis espécies de Triatoma (Lent \& Jurberg 1978). Trabalhos biológicos foram feitos sobre o tempo médio de alimentação de 26 min., sobre o número de defecações durante ou logo após o repasto entre dois e dez dias (Dias 1956), e sobre o período médio de duração do ciclo biológico que varia de 262 dias para os machos e 280 dias para as fêmeas em laboratório (Carcavallo et al. 1998, Canale et al. 1999). Poucos são os trabalhos sobre a sua biologia no habitat natural, embora no laboratório já existam alguns relatos (Silva 1985, Gonçalves et al. 1988, 2000) que detectaram um índice de $65,54 \%$ na infecção por $T$. cruzi para $T$. vitticeps. Aplicando a técnica de precipitina verificaram que participaram da dieta alimentar os seguintes vertebrados: tatu $30,3 \%$, humano/porco $13,1 \%$, ave/cão $11,5 \%$, cavalo $5,7 \%$, gambá $4,9 \%$, roedor $4,1 \%$ e boi $3,3 \%$. 


\section{MATERIAIS E MÉTODOS}

A equipe do laboratório capturou no quarto e sala de uma casa de pau-a-pique recoberta com telha de amianto 40 exemplares de T. vitticeps em vários estádios e 32 ovos. A repartição por estádio dos insetos era: 20 exemplares adultos, cinco ninfas de $5^{\circ}$ estádio, seis ninfas de $4^{\circ}$ estádio, duas ninfas de $3^{\circ}$ estádio, duas ninfas de $2^{\circ}$ estádio, cinco ninfas de $1^{\circ}$ estádio, além de seis ninfas de $2^{\circ}, 3^{\circ}, 4^{\circ}$ e $5^{\circ}$ estádio capturadas pela proprietária do imóvel. A análise do conteúdo do tubo digestivo dos triatomíneos foi realizada com a técnica de precipitina (Lorosa et al. 1998b). A bateria de antissoros e os respectivos títulos utilizados foram: anti-humano 1:15.000, ave 1:10.000, cão 1:15.000, gato 1:12.000, roedor 1:17.000, gambá 1:14.000 e boi 1: 15.000. A pesquisa da infecção natural por T. cruzi foi feita através de exame a fresco diretamente do conteúdo do tubo digestivo, que foi coletado em lâmina, por meio de uma leve pressão no abdome e levado à microscopia óptica. Coletamos sangue dos moradores da referida vivenda e os soros foram examinados pelos métodos de imunofluorescência indireta (IFI) e Elisa (EIE).

\section{RESULTADOS}

A análise do tubo digestivo dos triatomíneos realizada pela técnica de precipitina reforçou o seu ecletismo alimentar. Entre 46 triatomíneos (Tabela I), todos eram reativos e um total de 70 repastos sangüíneos foi identificado; 24 triatomíneos reativos $(52,2 \%)$ apresentavam repastos sangüíneos oriundos de uma só fonte, 18 de duas fontes $(39,1 \%)$ e quatro de três fontes $(8,7 \%)$. A identificação dos repastos mostrava que os humanos eram os hospedeiros predominantes $(31,4 \%)$, seguidos dos roedores $(24,3 \%)$, aves $(20 \%)$, cães $(15,7 \%)$ e marsupiais $(8,6 \%)$.

A taxa de infecção natural por T. cruzi foi de $13 \%$ (Tabela II). Um dos moradores da casa foi positivo para anticorpos anti-T. cruzi (teste de IFI com um título de 1/ $80)$.

\section{TABELA I}

Reações simples, duplas e triplas das fontes alimentares de Triatoma vitticeps (Stal, 1859) capturados em ambiente domiciliar em Arcádia, Miguel Pereira, RJ, Brasil

\begin{tabular}{lcc}
\hline Fonte de sangue & No. de repastos sangüíneos & $\%$ \\
\hline Humano & 22 & 31,4 \\
Roedor & 17 & 24,3 \\
Ave & 14 & 20 \\
Cão & 11 & 15,7 \\
Gambá & 6 & 8,6 \\
\hline Total & 70 & 100 \\
\hline
\end{tabular}

\section{DISCUSSÃO E CONCLUSÃO}

A freqüência que as espécies ditas secundárias vêm apresentando no intradomicílio, em várias regiões do Brasil, tem sido em grande escala.

Triatoma rubrofasciata De Geer, 1773, com índice de infecção natural de 16,9\%; participaram da sua dieta ali-
TABELA II

Índice de infecção natural por Trypanosoma cruzi em Triatoma vitticeps (Stal, 1859) segundo a fonte alimentar, capturados no ambiente domiciliar em Arcádia, Miguel Pereira, RJ, Brasil

\begin{tabular}{lcc}
\hline $\begin{array}{l}\text { Fontes } \\
\text { alimentares }\end{array}$ & $\begin{array}{c}\text { No. de } \\
\text { exemplares }\end{array}$ & $\begin{array}{c}\text { Positivo para } \\
\text { T. cruzi } \%\end{array}$ \\
\hline Humano & 2 & 4,4 \\
Roedor & 2 & 4,4 \\
Gambá & 1 & 2,2 \\
Cão/gambá & 1 & 2,2 \\
\hline Total & $6 / 46$ & 13 \\
\hline
\end{tabular}

mentar: roedor, cão, cavalo, gambá e humano na ilha de São Luís, Maranhão (Lorosa et al. 1998a).

Triatoma sordida (Stal, 1859), com infecção natural de $6,62 \%$; suas fontes alimentares foram ave, roedor e cão, no norte de Minas Gerais, Curvelo, Montes Claro e Januaria (Lorosa et al. 1998b).

Panstrongylus megistus Burneister, 1835, 70,6\% apresentavam-se infectados por T. cruzi e alimentando-se em ave, roedor e cão, no alto do Rio Paraná, Santa Catarina (Guilherme et al. 2001).

Triatoma costalimai Verano \& Galvão, 1959, com índice de $13,47 \%$ e as seguintes fontes alimentares: roedor, gambá, lagarto, cavalo, ave e tatu; Rhodnius neglectus Lent, 1954 com a infecção de 8,1\% alimentando-se em roedor, ave, gambá, cão e humano no norte do estado de Goiás (Lorosa et al. 1999d).

Triatoma rubrovaria Blanchard, 1843, espécie silvestre que atualmente evidencia progressiva adaptação à habitação humana e às dependências peridomicilaires, infectado por T. cruzi e alimentando-se em mamíferos e répteis encontrados nos estados do Paraná e Rio Grande do Sul (Almeida et al. 2000).

Triatoma brasiliensis Neiva, 1911 e Triatoma pseudomaculata Corrêa e Espínola, 1964, ambos de hábitos domésticos e peridomésticos, encontrados infectados com T. cruzi e alimentando-se em ave, cabra, cão, gato, roedor e humano (Alencar et al. 1977).

Dias et al. (1989) detectaram para o T. vitticeps um índice de $70,2 \%$ de infecção natural para fêmeas e 51,8\% para os machos; suas fontes alimentares foram humano, ave, roedor, marsupial, gato e cão no estado do Espírito Santo. Com esta mesma espécie, Gonçalves et al. (2000) encontraram um índice de infecção natural de $65,54 \%$ com a participação na dieta alimentar de tatu, porco, humano, ave, cão, cavalo, gambá, roedor e boi. O estudo foi realizado em duas áreas da localidade de Triunfo, $2^{\circ}$ distrito do município de Santa Maria Madalena, Rio de Janeiro.

O T. vitticeps, considerado até então como espécie silvestre, vem demonstrando capacidade de colonização no domicílio (Silveira et al. 1983, Diotaioti \& Dias 1987), tornando-se de interesse na epidemiologia da doença de Chagas.

Os resultados caracterizaram a domiciliação de $T$. vitticeps, com infecção para T. cruzi com um dos morado- 
res infectado, indicando a necessidade de realização de estudos sobre o comportamento dessa espécie e a sua relação com a transmissão do agente da doença de Chagas. É importante ressaltar seu ecletismo alimentar em situação intradomiciliar.

\section{AGRADECIMENTOS}

Ao Dr. François Noireau e ao Dr. Rodolfo U Carcavallo pelas sugestões e leitura crítica do texto e pela revisão do resumo em inglês.

\section{REFERÊNCIAS}

Alencar JE, Cunha RV, Araújo AGSC, Sobreira RTP 1977. Epidemiologia da doença de Chagas no Ceará. X - Hábitos alimentares dos vetores. Rev Soc Bras Med Trop XI: 45-53.

Almeida CE, Vinhaes MC, Almeida JR, Silveira AC, Costa J 2000. Monitoring the domiciliary and peridomiciliary invasion process of Triatoma rubrovaria in the state of Rio Grande do Sul, Brazil. Mem Inst Oswaldo Cruz 95: 761768.

Barros G, Mayrink W, Salgado AA, Barros RC, Sessa PA 1975. Contribuição para o conhecimento da doença de Chagas autóctone no estado do Espírito Santo. Rev Inst Med Trop São Paulo 17: 319-329.

Barros GC, Sessa PA, Barros RC, Matos EA 1980. Inquérito sorológico sobre doença de Chagas no banco de sangue do Hospital das Clínicas da Universidade Federal do Espírito Santo. Rev Pat Trop 9: 153-156.

Camargo ME, Silva GR, Castilho EA, Silveira AC 1984. Inquérito sorológico da prevalência de infecção chagásica no Brasil, 1975/1980. Rev Inst Med Trop São Paulo 26: $192-204$.

Canale DM, Jurgerg J, Carcavallo RU, Galvão C, Galíndez Girón I, Mena Segura CA, Rocha DS, Martinez 1999. A bionomia de algumas espécies. In RU Carcavallo, I Galíndez Girón, J Jurgerg, H Lent (eds), Atlas dos Vetores da Doença de Chagas nas Américas, Fiocruz, Rio de Janeiro, Vol. III, p. 839-890.

Carcavallo UR, Rocha DS, Galíndez Girón I, Sherlock I, Galvão C, Martinez A, Tonn JR, Corton E 1998. Fontes e padrões alimentares. In RU Carcavallo, I Galíndez Girón, J Jurgerg, H Lent (eds), Atlas dos Vetores da Doença de Chagas nas Américas, Fiocruz, Rio de Janeiro, Vol. II, p. 537-560.

Dias E 1956. Observações sobre alimentações defecções e tempo de sucção em alguns triatomíneos sul-americanos. Mem Inst Oswaldo Cruz 54: 115-125.

Dias JCP, Feitosa VR, Ferraz Filho AN, Rodrigues VLC, Alencar SA, Sessa PA 1989. Fonte alimentar e potencial vetorial de Triatoma vitticeps (Stal, 1859) com relação à doença de Chagas humana no estado do Espírito Santo, Brasil (Hemiptera, Reduviidae). Mem Inst Oswaldo Cruz 84: 165-173.

Diotaiuti L, Dias JCP 1987. Estudo comparativo do ciclo evolutivo de Rhodnius neglectus alimentados em pombos ou camundongos. Rev Soc Bras Med Trop 20: 95-100.

Gonçalves TCM, Oliveira E, Dias LS, D'Almeida M, Nogueira WO, Pires FA 1998. An investigation on the ecology of Triatoma vitticeps (Stal. 1859) and its possible role in the transmission of Trypanosoma cruzi, in the locality of Triunfo, Santa Maria Madalena municipal district, state of Rio de Janeiro. Mem Inst Oswaldo Cruz 93: 711-717.

Gonçalves TCM, Victorio VMN, Jurberg J, Cunha V 1988.
Biologia do Triatoma vitticeps (Stal, 1859) em condições de laboratório (Hemiptera: Reduviidae: Triatominae). I ciclo evolutivo. Mem Inst Oswaldo Cruz 83: 519-523.

Gonçalves TCM, Rocha DC, Cunha RA 2000. Feeding patterns of Triatoma vitticeps in the state of Rio de Janeiro Brazil. Rev Saúde Públ 34: 384-52.

Guilherme ALF, Lorosa ES, Costa AL, Pavanelli GC, Araújo SM 2001. Panstrongylus megistus em ecótopos artificiais de ilhas do Alto Rio Paraná. Rev Soc Bras Med Trop 34: 491-494.

Jurgerg J, Campos P 1995. Morfologia de huevos e ninfas de Triatoma vitticeps Stal, 1859 (Hemiptera - Reduviidae Triatominae). Entomol Vect 2: 9-22.

Lent H 1942. Transmissores da moléstia de Chagas no estado do Rio de Janeiro. Rev Flum Med 6: 1-13.

Lent H, Jurberg J 1978. Estudo comparativo da genitália externa masculina de seis espécies de Triatoma Laporte, 1832 que mais freqüentemente habitam o domicílio humano (Hemiptera - Reduviidae). Rev Brasil Biol 38: 931-944.

Lent H, Wygodzinsky P 1979. Revision the Triatominae (Hemiptera, Reduviidae) and their significance as vectors of Chagas' disease. Bull Amer Museum Nat Hist 163: 127516.

Lorosa ES, Andrade RE, Serra MGA, Rebelo JMM, Vinhaes MC 1998a. Estudo das fontes alimentares através da reação de precipitina e grau de infectividade em Triatoma rubrofasciata (De Geer, 1773) coletados na Ilha de São Luiz, Maranhão. Entomol Vect 5: 241-250.

Lorosa ES, Andrade RE, Santos SM, Pereira CA 1998b. Estudo da infecção natural e da fonte alimentar do Triatoma sordida, (Stal, 1859), (Hemiptera - Reduviidae) na região norte de Minas Gerais, Brasil, através da reação de precipitina. Entomol Vect 5: 13-22.

Lorosa ES, Andrade RE, Santos SM, Pereira CA, Vinhaes MC, Jurberg J 1999. Estudo da infecção natural e fontes alimentares de Triatoma costalimai Verano \& Galvão, 1959, Rhodnius neglectus Lent, 1954 e Psammolestes tertius Lent \& Jurberg, 1965 do estado de Goiás, Brasil, através da técnica de precipitina. Entomol Vect 6: 405-414.

Pinto AFS, Santos UM, Zaganelli FL, Almeida AZ, Carrancho PV 1969. Doença de Chagas no estado do Espírito Santo VI - Observações sobre o Triatoma vitticeps, vetor do Trypanosoma tipo cruzi no município de Alfredo Chaves ES. Rev Bras Med Trop 3: 53-54.

Pinto LFS, Vieira NER, Milanez MC, Pereira FEL, Sessa PA 1986. Doença de Chagas autóctone no Espírito Santo: relato de caso agudo em gestante com transmissão para o feto. Rev Soc Brasil Med Trop 19 (Supl.): 78.

Santos UM, Murad V, Chapadeiro E, Lima FEP 1969. Doença de Chagas no estado do Espírito Santo. III - Vetores do Trypanosoma. Rev Bras Med Trop 3: 51-52.

Sessa PA, Carias VDR 1986. Infecção natural de triatomíneos do Espírito Santo por fragelados morfologicamente semelhantes ao Trypanosoma cruzi. Rev Soc Brasil Med Trop 19: 99-100.

Silva IG 1985. Influência da Temperatura na Biologia de 18 Espécies de Triatomíneos (Hemiptera: Reduviidae) e no Xenodiagnostico, MSc Thesis, Universidade Federal do Paraná, Curitiba, 169 pp.

Silveira AC, Alencar TA, Máximo MH 1983. Sobre o Triatoma vitticeps Stal, 1859, no estado do Espírito Santo, Brasil. Resumos de comunicações da X Reunião Anual de Pesquisa Básica em Doença de Chagas, Caxambu, MG, 5-8. 